\title{
SDN Coordination for CCN and FC Content Dissemination in VANETs
}

\author{
Ridha Soua $^{1}$, Eirini Kalogeiton ${ }^{2}$, Gaetano Manzo $^{2,3}$, Joao M. Duarte ${ }^{2,4}$, Maria Rita \\ Palattella $^{1}$, Antonio Di Maio ${ }^{1}$, Torsten Braun ${ }^{2}$, Thomas Engel ${ }^{1}$, Leandro A. Villas ${ }^{4}$, \\ and Gianluca A. Rizzo ${ }^{3}$ \\ 1 SnT, University of Luxembourg, Luxembourg \\ \{ridha.soua, maria-rita.palattella, antonio.dimaio, thomas.engel\} @ \\ uni.lu \\ 2 University of Bern, Switzerland \\ \{kalogeiton, duarte, braun\} @inf.unibe.ch \\ 3 HES-SO, Switzerland \\ \{gaetano.manzo, gianluca.rizzo\}@hevs.ch \\ 4 Institute of Computing, University of Campinas, Brazil \\ leandrodic.unicamp.br
}

\begin{abstract}
Content dissemination in Vehicular Ad-hoc Networks has a myriad of applications, ranging from advertising and parking notifications, to traffic and emergency warnings. This heterogeneity requires optimizing content storing, retrieval and forwarding among vehicles to deliver data with short latency and without jeopardizing network resources. In this paper, for a few reference scenarios, we illustrate how approaches that combine Content Centric Networking $(\mathrm{CCN})$ and Floating Content (FC) enable new and efficient solutions to this issue. Moreover, we describe how a network architecture based on Software Defined Networking (SDN) can support both $\mathrm{CCN}$ and FC by coordinating distributed caching strategies, by optimizing the packet forwarding process and the availability of floating data items. For each scenario analyzed, we highlight the main research challenges open, and we describe a few possible solutions.
\end{abstract}

Key words: VANETs, Software Defined Networking, Content Centric Networking, Floating Content, Content Caching and Replication

\section{Introduction}

Vehicular Ad-hoc Networks (VANETs) [1] allow communications among vehicles and between vehicles and fixed infrastructure, aiming to support a wide range of services and applications to make travel experience pleasant, safe, and informed [2]. Applications envisioned for VANETs vary from traffic conditions and accident warnings, to infotainment services such as live video streaming, live gaming, etc. The main technical challenges in VANET communications are related to the high dynamicity and volatility of the vehicular environment. Therefore, mechanisms for online adaptation of the network configuration to the wireless medium, to highly varying inter-user distance and to node density, etc. are required [1]. Though a lot of work has been done in proposing mechanisms for efficient content dissemination, a lot of work remains to be done 
to reliably support infotainment applications with acceptable Quality of Service (QoS) and Quality of Experience (QoE).

This paper describes a possible approach to tackle this issue, based on combining three paradigms: Floating Content (FC), Software-Defined Networking (SDN), and Content-Centric Networking (CCN). Content-Centric Networking [3] allows messages to be exchanged throughout the network based on their content and not on the location of the hosts. In settings characterized by high node mobility and volatility, such as VANETs, this may greatly increase the chance of delivering the requested content in case of disrupted links and frequent changes of network topologies. However, mechanisms are required to adapt routing decisions to such changes and to achieve a good balance between optimizing content delivery likelihood and resource utilization.

In warning applications, data packets are usually small and can be disseminated between vehicles and between vehicles and infrastructure such as Road Site Units (RSUs) without significantly affecting the available communication bandwidth. For these types of services, the push-based communication approach, usually adopted in publish/subscribe applications, is well suitable. Therefore, Floating Content, an opportunistic communication scheme, which supports infrastructure-less distributed content sharing over a given geographic area, could be a good candidate for the implementation of these services. The basic formulation of FC is push-based and conceived to suit settings, in which a large fraction of nodes in a given area (the Anchor Zone, AZ) are interested in receiving small messages $[4,5]$. The idea behind FC is to store a given content object in a spatial region without any fixed infrastructure, making it available through opportunistic communications to all users traversing that region. Whenever a node possessing the content object is within the transmission range of some other nodes not having it, the content object is replicated. When a node with the content object moves out of its spatio-temporal limits, it deletes the content object. The content object may be available on a set of nodes and moves over time within the AZ, even after the node that has originally generated the content has left the AZ. Thus, the content object 'floats' within the AZ. Besides the advantages that both FC and CCN may bring in storing and disseminating content in VANETs, their performance could be improved by the coordination of a centralized entity that has global knowledge of the nodes, mobility, their interest in a given content, as well as other information. It can properly set FC and $\mathrm{CCN}$ parameters (e.g., AZ size, time to replicate content, strategy for content caching) and select which approach is more suitable for a given use case [6].

Initially designed for wired networks, Software Defined Networking (SDN) has been recognized as an attractive and promising approach for wireless and mobile networks too [7]. These networks can benefit from the flexibility, programmability and centralized control view offered by SDN, under different aspects, such as wireless resource optimization (i.e., channel allocation, interference avoidance), packet routing and forwarding in multi-hop multi-path scenarios as well as efficient mobility and network heterogeneity management. The pioneering SDN-based architecture for VANETs was proposed by $\mathrm{Ku}$ et al. [8], and afterwards enhanced [9, 10] by adding cloud and fog computing components. In a scenario with a variety of vehicular services with very diverse communication requirements, SDN holds the potential to improve the management of content delivery [11]. A first Type-Based Content Distribution (TBCD) method 
was proposed in [12] to improve content caching and forwarding in a SDN-enabled VANET. TBCD adopts a push-and-pull approach for delivering content, based on the type of content, and on the number of users interested in it.

In line with that, we investigate how SDN could be beneficial in VANETs for improving CCN-based content caching and FC-based content replication within a given geographical area. The rest of the paper is organized as follows. Section 2 first describes how CCN and FC can be applied in vehicular network scenarios. Section 3 envisages the research areas where the use of a SDN controller may help improving the performance of CCN in vehicular networks. Section 4 illustrates how FC can benefit from some form of coordination among nodes, when implemented through a SDN controller. Finally, Section 5 concludes the paper.

\section{Content Dissemination in VANETs}

In this section, we discuss how to support content dissemination in highly mobile VANETs using CCN and FC. Some related works already proposed CCN modifications to better suit the paradigm to VANET features. Goals were to decrease packet forwarding load and dealing with difficulties in maintaining Forwarding Information Base (FIB) entries. In [13], an additional field was introduced in the Data message to help requesters in selecting the best content provider. To increase content availability, authors in [14] propose that each vehicle caches all received Data and forwards all Interest messages to all available interfaces. However, increasing the message size and forwarding multiple Interest messages for given content increase traffic load. Therefore, new approaches are needed.

When content objects are bound to a specific geographic region, and stored on all (or a subset of) nodes within the region itself, FC could play a key role for improving CCN. By replicating content, FC introduces redundancy, which can be useful when nodes cannot be continuously active and hence do not make content permanently available. Reasons for this could be high node failure rates, intermittent connectivity, load balancing, or energy management involving node duty cycling. Moreover, floating content can make searching and finding content more efficient, if the geographic area of the floating content can be derived from the content name. Normally, to retrieve content, requesters issue Interest messages including a content name describing the requested content. To ensure that the content bound to a geographic area is found, the content name could be mapped to that area. Consequently, an Interest message is forwarded to that geographic area to meet the requested content. Mapping can be supported by either having a geographic ID in the content name or by having an intermediate node, which translates the original content name into a geographic name. After the Interest has met a content source, the Data message including the content is sent back to the requester based on the stored information in the PIT.

\subsection{Receiver and Source Mobility Support}

In VANETs, both source and requester (receiver) nodes might become mobile. Although CCN has implicit support for receiver mobility, certain problems can occur in 
VANETs in case of very high receiver speeds [15]. If both the RTT of Interest / Data messages and the velocity of the mobile requester are rather low, then standard CCN based on FIB and PIT forwarding will work, because returned Data messages will meet valid PIT entries. However, in case of longer RTTs and fast mobile receivers, PIT entries might be invalid for returning Data messages and the content cannot be delivered.

Receiver mobility can be supported by FC. An Interest could be sent towards the source. In the Interest message an area could be indicated, specifying where the content shall be sent (e.g., the area that the receiver will visit soon). The Data is then sent to the indicated area, where it might float. The receiver can then pick it up when it arrives at the indicated area. However, this approach somehow contradicts the fundamentals of $\mathrm{CCN}$, since Data messages are not sent along the reverse path (considering Interests) using the information stored in PITs.

Source mobility, not natively supported by $\mathrm{CCN}$, makes content search more difficult. Source mobility either requires frequent content advertisement updates or frequent content searches possibly causing significant overhead by broadcasts. It can be supported efficiently by FC if content is assigned to a certain geographic area. If it is ensured that always some nodes storing the content are within the assigned area, then the content can be provided by at least one of the nodes independent on source mobility.

\subsection{Agent-Based Content Retrieval (ABCR)}

Another approach for requesting and receiving content is based on using agents. Requesters can delegate content retrieval to one or more agents [16] and retrieve from them the content later. A requester broadcasts an Interest to potential agents, which respond by Data messages describing their offer to retrieve content on behalf the requester. The requester then confirms the agent selection. Later, when the requester meets the agent again, it can retrieve the content using traditional CCN message exchange.

In a VANET, a user might like to retrieve a content object from a location that is not in range, and where the vehicle is not scheduled to go. By letting requester and agent know their respective typical or expected paths, the content source could select the right agent (the one with the right path) for sending back the content object. Here we assume that the content source knows the path of the requester and of the candidate agents for carrying the content and that the content source will give information about the path of the requester to the agent carrying the content. Uncertainty in space and time can make the rendezvous hard to implement. To face this issue, the agent carrying the content could implement an AZ, in that the desired content floats and that the requester will traverse with high likelihood.

Agents can also be used to come up with a solution for receiver mobility without the need to modify $\mathrm{CCN}$. To address receiver mobility, a mobile requester intending to visit an indicated area can delegate content retrieval to an agent located in the indicated area. The agent requests the information from the content source and disseminates it using FC mechanisms in the indicated area. The mobile receiver then picks up the content from the agent or from another FC node when visiting the indicated area. 


\subsection{FC-assisted, Geographic Content Centric (DTN) Routing}

In settings where volatility makes it unfeasible or inefficient to maintain a path between content requester(s) and content source, FC may be used to support so-called geographic content centric routing. We assume that all nodes and content objects can be identified unequivocally, and that nodes know their location and future trajectory with some uncertainty. Such knowledge could be derived from past spatio-temporal patterns. By letting each node share through FC its own data on network state (e.g., node positions and trajectories, node content), FC can be used to build at each node a common, shared representation of the network and its evolution over time. Such representation could be used to implement various strategies for Delay Tolerant Network (DTN) routing.

As a complement, when nodes are sparse and/or their mobility does not allow to build a stable path for content dissemination, Interest packets could be made to float in an area around the originator node. The floating content would be used as a "fishing net" to improve the chance of finding content among passing nodes. As a result, each node within an area would then maintain and share a table (i.e., floating PIT), describing a list of requested content objects. For each requested content object, the table stores a list of requesters. When meeting a node with one or more of the requested content objects, those could be forwarded to the requesters using some form of DTN routing scheme, possibly using the floating network state.

\section{SDN Support for CCN in VANETs}

\subsection{SDN for CCN Forwarding and Broadcasting}

In large-scale networks the size of FIB tables on each $\mathrm{CCN}$ node can easily increase due to the large amount of exchanged content and the large number of content sources. Alternatively, the CCN routers that request content broadcast Interest packets to neighbor nodes in order to receive it. Subsequently, the possibility of congestion in paths through the network can be very high. There have been several attempts to encounter these problems; some of them try to identify a path between a requester and a content source and store this path for future references [17, 18]. Others propose to forward Interests based on the distance between sender and receiver [19]. A similar approach has been adopted also in CCVN [13], where messages are broadcast in the entire VANET, but in case of collisions they are re-broadcast according to the distance from previous senders. To improve content retrieval, in [20] authors suggest to utilize the different interfaces of a CCN node to transmit Interests simultaneously through multiple interfaces. For the same aim, Udugama et al. [21] propose to split the Interest messages of the same request and send them through multiple paths at the same time.

A SDN controller, with its centralized view of the network, can support the design of more efficient $\mathrm{CCN}$ forwarding strategies. For instance, a controller can be responsible for selecting unicast paths between requester and content source in a given cluster of the network [22]. To integrate the SDN approach in CCN networks, Charpinel et al. [23] proposed a CCN controller that has a complete view of the network. It defines 
the forwarding strategy, and installs forwarding rules in the FIBs of CCN routers. In every CCN router there is a Cache Rules Table (CRT). The CCN controller sends cache rules (replacement policies) to the $\mathrm{CCN}$ switches, which are stored in the CRT table. The CCN router caches the content according to the rules that exist in the CRT table. The presence of a centralized controller could be further exploited to improve CCN performance. For instance, a SDN controller can determine the number of chunks to transmit through a face and send them through multiple faces simultaneously [24].

Our Approach CCN content discovery mechanisms can be improved by integrating SDN logic. A SDN controller could setup a path between a requester and a content source and install forwarding rules in the FIB tables of the nodes [23]. Furthermore, flows could be set up similar to the Dynamic Unicast approach [17]. Then a SDN controller could guarantee the QoS for each flow and could transmit the message through the best flow. Moreover, as shown in Fig.1(a), SDN could support the set up of multipath communication. When a vehicle sends or receives a message (Interest or Data), it could send (or receive) it through multiple (redundant) paths. A SDN controller could discover several paths that satisfy Interest messages of the same request. Then, the controller could send Interest messages through those paths in parallel, in order to retrieve Data much faster.

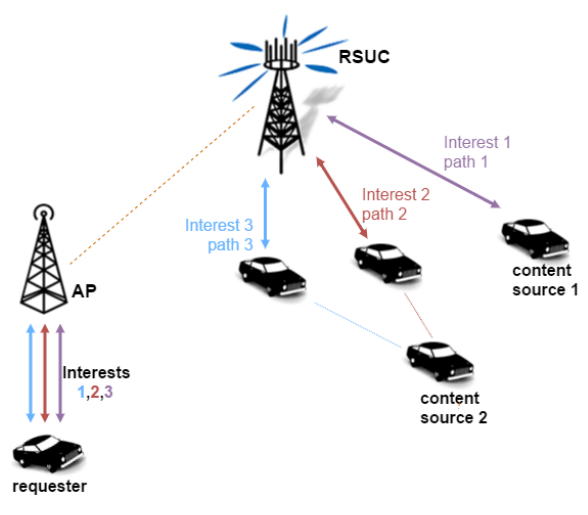

(a)

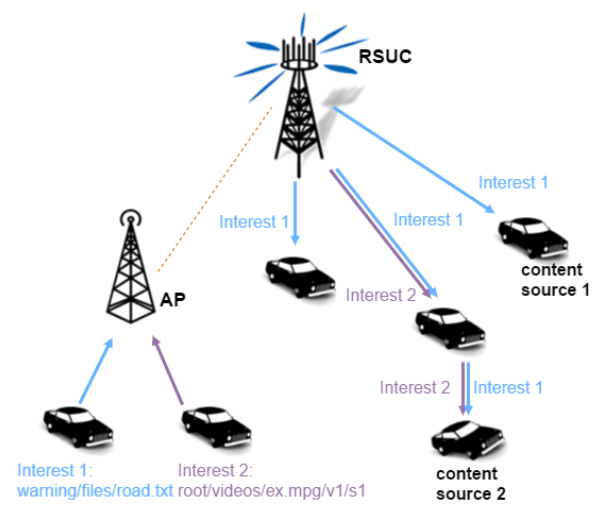

(b)

Fig. 1. SDN support for CCN forwarding in VANETs: (a) Forwarding of Interests along different paths, (b) Different forwarding strategy based on Interests names

In a CCN-based VANET, several applications will generate requests with different names. The network must treat these requests in a different way. For instance, a safety application generating warning messages should forward these messages to the entire network. On the contrary, an entertainment application sending video requests should forward these messages through an appropriate face in order to find a content source. A SDN controller could install such forwarding rules to make sure that each message is treated differently, according to its name (Fig.1(b)). 


\subsection{SDN for CCN Caching}

Caching techniques are used to avoid accessing the server storing the original copy for content requests. Specifically, the buffer memory in vehicles is considered as a cache space for replicas. Indeed, upon a cache hit at a Content Store, the Data message is sent back to the vehicle that sent the Interest. In case of a cache miss, the Interest message shall be forwarded according to a name-based routing strategy, if a similar request is not already pending. Caching enhances content discovery, retrieval and delivery in VANETs by providing multiple sources (caches) of the content. However, the explosion of infotainment applications with their ubiquitous replicas creates an increasing demand for the scarce spectrum in VANETs. Basically, caching is coupled with two fundamental questions: what content to cache and where to cache it.

Caching all content along the delivery paths as suggested in [25] may cause serious performance degradation. Even if the cache size is not a major concern in VANETs, it is not obvious if this cache can keep up the pace with the increasing scale of multimedia content distribution over VANETs. For these reasons, it was proposed in [26, 27, 28] to cache only popular content (i.e., content which has been requested a number of time equal or larger than a fixed Popularity Threshold, PT). Other works rely on the user interest for deciding what content to keep [29], i.e., nodes will cache only the content they are interested in.

Concerning where to cache, one trivial solution is to cache replicas in every vehicle [30]. The short-lived nature of links in VANETs, coupled with the time and spacerelevant nature of content, accentuates the concern about the selection of relevant nodes that can cache specific content. Therefore, most new caching schemes attempt to reduce the nodes' caching redundancy by only selecting a subset of nodes in the delivery path. This subset of nodes has high probability to get a cache hit and hence nodes in this subset are called "central" nodes. Centrality-based solutions for node selection were proposed in the context of static networks. However, their extension to highly mobile networks, such as VANETs, is a thorny problem as centrality is not trivial [31]. Recent works [32,33] focus on the social aspect of caching. Users in the same social space are likely to request the same content. Hence, the content is proactively pushed to the cache of users' proximate neighbors. The definition of these proximate neighbors is challenging. While in [32] proximate nodes are 1 - hop neighbors, authors of [33] define these nodes as the nodes having a specific ratio of common content items with the user.

Our Approach Given the unique features of VANETs, SDN can provide centralized cached content management. A SDN controller could instruct Content Providers (CP) to trigger on-demand caching when popular content has been identified. In the same way, the SDN controller could fix the value of the PT according to different metrics. Moreover, the SDN controller, being the owner of the caching logic, will determine which vehicles (Content Cachers) should store replicas of the popular content at each period of time. This centralized caching logic helps authorities to program and deploy the desired caching behavior.

Figure 2 illustrates an example of our proposed SDN-based caching approach in VANETs. Two RSUs are orchestrated by one RSU Controller (RSUC). Each RSU is covering a specific geographical area on the highway. The RSUC fixes the PT and the 


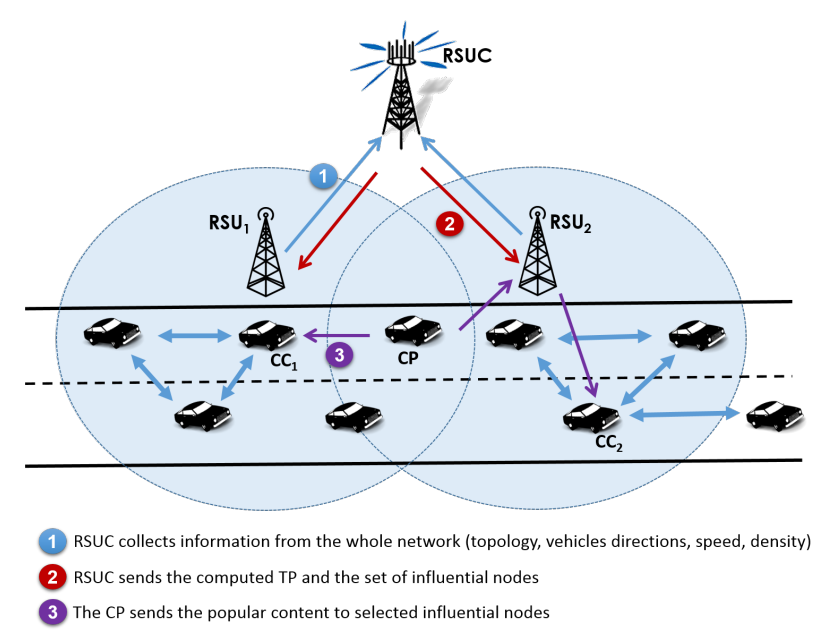

Fig. 2. SDN-based caching approach in VANETs.

set of influential vehicles [34] in the zone. The set of influential nodes is time-variant since vehicles can enter and leave the network frequently. Therefore, the RSUC should periodically (or any time is needed) notify vehicles in the network about the updated set of influential nodes. Once the number of requests for the content produced by the $\mathrm{CP}$, reaches the PT, the RSUC will trigger its caching spreading strategy. The popular content will not be sent to the immediate 1-hop neighbors of CP like in [32], but will be sent to the influential vehicles $\left(C C_{1}\right.$ and $\left.C C_{2}\right)$. This mechanism avoids overloading the network with replicas of the same content. The $\mathrm{CP}$ is aware of the set of influential nodes and send them accordingly the replicas. While $C C_{1}$ is a 1-hop neighbor of the $\mathrm{CP}, C C_{2}$ is out of its transmission range. Therefore, the content is sent first to $R S U_{2}$ and then $R S U_{2}$ forwards the popular content to $C C_{2}$. Vehicles interested in the content of $\mathrm{CP}$ can ask $C C_{1}$ or $C C_{2}$ to retrieve it. Thus, vehicles far away from the $\mathrm{CP}$ (the node that generated the original content) can get a replicas by asking the nearest $C C$.

\section{SDN support for FC in VANETs}

In FC, information dissemination is geographically limited by the AZ. However, there is no rule on how a user defines the geographic origin, radius and expiration time of the AZ. Simulation results [35] show that the ratio between AZ radius and communication range has an impact on information availability. Besides, authors of [4], provide the criticality condition under which a population of mobile nodes can support the floating content. This condition is related to node density, transmission range, and AZ. Hence, defining the AZ size and shape is a complex task due to vehicles' high mobility and most importantly the lack of centralized network administration.

Furthermore, the main goal of FC is to ensure that each node inside the AZ gets the content and replicates it through opportunistic message exchanges. This exchange can 
involve a huge number of vehicles (especially in urban areas) and a large amount of information. A majority of studies $[4,5,35]$ assume that when a node with a piece of content in the AZ comes within the transmission range of some other node that does not have it, the piece of content is replicated. This replication takes into account neither the popularity of the content nor the size of set of nodes carrying the content. Unfortunately, more information is not always better, at least not in VANETs, when a massive number of messages increase both spectrum congestion and management challenges.

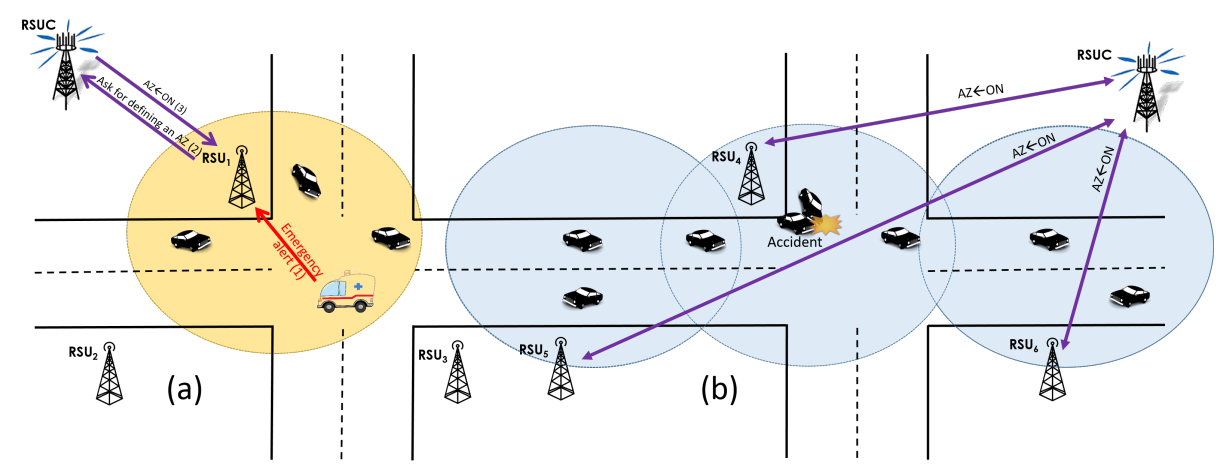

Fig. 3. (a) SDN-based FC activation, (b) AZ reshaping in an accident intersection scenario

Our Approach The centralized intelligence and configuration flexibility that SDN offers allows vehicles to flexibly define the AZ size and shape to cope with content deletion. In challenging environments with highly varying density, SDN can ensure better persistence and availability of floating content by accurately tuning the AZ size. Once an AZ has been defined, the RSUC could reshape the existing AZ or trigger the activation of a new AZ based on its global knowledge. This on-demand creation or extension of an AZ saves network resources. Figure 3(a) shows a use case where an AZ is created upon demand. An emergency vehicle enters the zone covered by $R S U_{1}$ where there is no pre-established AZ. The emergency vehicle asks $R S U_{1}$ to define an AZ via V2I communication. Then the request is forwarded by $R S U_{1}$ to the RSUC, which sends back the parameters of the AZ (shape, size, lifetime) based on the content lifetime, nodes density, and mobility. Since communication in FC is infrastructure-less, this temporary on-demand AZ creation was not possible without using SDN. Moreover, SDN could be involved in reshaping of an existing AZ. One interesting use case is information dissemination in an intersection, which is a highly localized area as depicted in Figure 3(b). To ensure that the content is floating outside the AZ defined by the intersection, one possible solution would be to reshape the AZ and make it bigger. However, as argued in [5], the bigger the AZ size is, the lower is the success probability (i.e. probability for a node entering in AZ to get the content), if node transmission ranges and density are fixed. Hence, the RSUC will define a new AZ's shape based on its global knowledge of the geographical area, node's space distribution, RSUs' traffic load, and the desired size of the floating zone. 
Content replication could also be supported by SDN. The RSUC can define, through information collected from RSUs, the floating content's priority. Subsequently, a node can decide to receive and/or ignore content based on a priority rule that can take into account the content lifetime, number of nodes replicating the content, and the intermittent nature of the connections. Thus, the SDN controller implemented in RSUC could figure out the content popularity and decide with which frequency the content is replicated. The RSUC can also define a Range of Interest (ROI) inside the AZ. Indeed, the content is replicated mainly inside the ROI (resp. outside in other applications). For instance, if there are not so many copies of a content object inside the ROI, the SDN controller could allow the exchange of content even outside (resp. inside) the ROI's coverage range. Consequently, the probability of loosing the content is very small.

\section{Conclusions}

In this work, we have identified $\mathrm{CCN}$ and $\mathrm{FC}$ as two suitable enablers for improving content storing, dissemination, and forwarding in future VANETs. Both should be adapted to the high dynamic and volatile network environment. To this aim, SDN could provide support for $\mathrm{CCN}$ and FC, e.g., to select, according to node mobility, the influential nodes where the content should be cached, or to activate the $\mathrm{AZ}$ where the content should float. Future work will investigate the envisioned integration of CCN, FC and SDN in VANETs in more depth.

\section{Acknowledgment}

This work was undertaken under the CONTACT project, CORE/SWISS/15/IS/10487418, funded by the National Research Fund Luxembourg (FNR) and the Swiss National Science Foundation (SNSF) project no. 164205. The authors also like to thank the São Paulo Research Foundation (FAPESP) for the financial support by grant 2016/09254-3.

\section{References}

1. Saif Al-Sultan, Moath M Al-Doori, Ali H Al-Bayatti, and Hussien Zedan. A comprehensive survey on vehicular ad hoc network. Journal of network and computer applications, 37:380 392, 2014.

2. Felipe Domingos Da Cunha, Azzedine Boukerche, Leandro Villas, Aline Carneiro Viana, and Antonio AF Loureiro. Data communication in VANETs: a survey, challenges and applications. PhD thesis, INRIA Saclay; INRIA, 2014.

3. Van Jacobson, Diana K Smetters, James D Thornton, Michael F Plass, Nicholas H Briggs, and Rebecca L Braynard. Networking named content. In Proceedings of the 5th international conference on Emerging networking experiments and technologies, pages 1-12. ACM, 2009.

4. E. Hyyti, J. Virtamo, P. Lassila, J. Kangasharju, and J. Ott. When does content float? characterizing availability of anchored information in opportunistic content sharing. In INFOCOM, 2011 Proceedings IEEE, pages 3137-3145, April 2011. 
5. S. Ali, G. Rizzo, V. Mancuso, and M. A. Marsan. Persistence and availability of floating content in a campus environment. In 2015 IEEE Conference on Computer Communications (INFOCOM), pages 2326-2334, April 2015.

6. G. Rizzo, M. R. Palattella, T. Braun, and T. Engel. Content and context aware strategies for qos support in vanets. In 2016 IEEE 30th International Conference on Advanced Information Networking and Applications (AINA), pages 717-723, March 2016.

7. Mao Yang, Yong Li, Depeng Jin, Lieguang Zeng, Xin Wu, and Athanasios V Vasilakos. Software-defined and virtualized future mobile and wireless networks: a survey. Mobile Networks and Applications, 20(1):4-18, 2014.

8. Ian $\mathrm{Ku}$, You Lu, Mario Gerla, Rafael Gomes, Francesco Ongaro, and Eduardo Cerqueira. Towards software-defined vanet: Architecture and service. Conference: Annual Mediterranean Ad Hoc Networking Workshop MEDHOCNET, 2014.

9. Mohammad Ali Salahuddin, Ala Al-Fuqaha, and Mohsen Guizani. Software-defined networking for rsu clouds in support of the internet of vehicles. Internet of Things Journal, IEEE, 2(2):133-144, 2015.

10. Nguyen B Truong, Gyu Myoung Lee, and Yacine Ghamri-Doudane. Software defined networking-based vehicular adhoc network with fog computing. In Integrated Network Management (IM), 2015 Conference. IFIP/IEEE, 2015.

11. Matthew Broadbent, Daniel King, Sean Baildon, Nektarios Georgalas, and Nicholas Race. Opencache: A software-defined content caching platform. In Network Softwarization (NetSoft), 2015 1st IEEE Conference on, pages 1-5. IEEE, 2015.

12. Yi Cao, Jinhua Guo, and Yue Wu. Sdn enabled content distribution in vehicular networks. In Innovative Computing Technology (INTECH), 2014 Fourth International Conference on, pages 164-169. IEEE, 2014.

13. Marica Amadeo, Claudia Campolo, and Antonella Molinaro. Enhancing content-centric networking for vehicular environments. Computer Networks, 57(16):3222-3234, 2013.

14. Giulio Grassi, Davide Pesavento, Giovanni Pau, Rama Vuyyuru, Ryuji Wakikawa, and Lixia Zhang. Vanet via named data networking. In Computer Communications Workshops (INFOCOM WKSHPS), 2014 IEEE Conference on, pages 410-415. IEEE, 2014.

15. Carlos Anastasiades, Torsten Braun, and Vasilios A Siris. Information-centric networking in mobile and opportunistic networks. In Wireless Networking for Moving Objects, pages 14-30. Springer, 2014.

16. Carlos Anastasiades, Tobias Schmid, Jürg Weber, and Torsten Braun. Information-centric content retrieval for delay-tolerant networks. Computer Networks, 2016.

17. Carlos Anastasiades, Jürg Weber, and Torsten Braun. Dynamic unicast: Information-centric multi-hop routing for mobile ad-hoc networks. Computer Networks, 2016.

18. Emmanuel Baccelli, Christian Mehlis, Oliver Hahm, Thomas Schmidt, and Matthias Wählisch. Information centric networking in the iot: Experiments with ndn in the wild. In 1st ACM Conference on Information-Centric Networking (ICN-2014). ACM, 2014.

19. Giulio Grassi, Davide Pesavento, Giovanni Pau, Lixia Zhang, and Serge Fdida. Navigo: Interest forwarding by geolocations in vehicular named data networking. In World of Wireless, Mobile and Multimedia Networks (WoWMoM), 2015 IEEE 16th International Symposium on a, pages 1-10. IEEE, 2015.

20. Cheng Yi, Alexander Afanasyev, Lan Wang, Beichuan Zhang, and Lixia Zhang. Adaptive forwarding in named data networking. ACM SIGCOMM computer communication review, 42(3):62-67, 2012.

21. Asanga Udugama, Xinyi Zhang, Koojana Kuladinithi, and Carmelita Goerg. An on-demand multi-path interest forwarding strategy for content retrievals in ccn. In 2014 IEEE Network Operations and Management Symposium (NOMS), pages 1-6. IEEE, 2014. 
22. Jaehyeok Son, DoHyeon Kim, Hyo Sung Kang, and Choong Seon Hong. Forwarding strategy on sdn-based content centric network for efficient content delivery. In 2016 International Conference on Information Networking (ICOIN), pages 220-225. IEEE, 2016.

23. Sergio Charpinel, Celso Alberto Saibel Santos, Alex Borges Vieira, Rodolfo Villaca, and Magnos Martinello. Sdccn: A novel software defined content-centric networking approach. In 2016 IEEE 30th International Conference on Advanced Information Networking and Applications (AINA), pages 87-94. IEEE, 2016.

24. Doo Ho Lee, Kyi Thar, DoHyeon Kim, and Choong Seon Hong. Efficient parallel multi-path interest forwarding for mobile user in ccn. In 2016 International Conference on Information Networking (ICOIN), pages 390-394. IEEE, 2016.

25. Y. T. Yu and M. Gerla. Information-centric vanets: A study of content routing design alternatives. In 2016 International Conference on Computing, Networking and Communications (ICNC), pages 1-5, Feb 2016.

26. C. Bernardini, T. Silverston, and O. Festor. Mpc: Popularity-based caching strategy for content centric networks. In 2013 IEEE International Conference on Communications (ICC), pages 3619-3623, June 2013.

27. S. B. Lee, S. H. Y. Wong, K. W. Lee, and S. Lu. Content management in a mobile ad hoc network: Beyond opportunistic strategy. In INFOCOM, 2011 Proceedings IEEE, pages 266-270, April 2011.

28. Y. Xu, Shuai Ma, Y. Li, F. Chen, and S. Ci. P-cls: A popularity-driven caching location and searching scheme in content centric networking. In 2015 IEEE 34th International Performance Computing and Communications Conference (IPCCC), pages 1-8, Dec 2015.

29. J. Iqbal and P. Giaccone. Interest-based cooperative caching in multi-hop wireless networks. In 2013 IEEE Globecom Workshops (GC Wkshps), pages 617-622, Dec 2013.

30. Lucas Wang, Alexander Afanasyev, Romain Kuntz, Rama Vuyyuru, Ryuji Wakikawa, and Lixia Zhang. Rapid traffic information dissemination using named data. In Proceedings of the 1st ACM Workshop on Emerging Name-Oriented Mobile Networking Design - Architecture, Algorithms, and Applications, NoM '12, pages 7-12, New York, NY, USA, 2012. ACM.

31. L. Gallos, S. Havlin, M. Kitsak, F. Liljeros, H. Makse, L. Muchnik, and H. Stanley. Identification of influential spreaders in complex networks. Nature Physics, 6(11):888-893, Aug 2010.

32. C. Bernardini, T. Silverston, and O. Festor. Socially-aware caching strategy for content centric networking. In Networking Conference, 2014 IFIP, pages 1-9, June 2014.

33. G. Moualla, P. A. Frangoudis, Y. Hadjadj-Aoul, and S. Ait-Chellouche. A bloom-filter-based socially aware scheme for content replication in mobile ad hoc networks. In 2016 13th IEEE Annual Consumer Communications Networking Conference (CCNC), pages 359-365, Jan 2016.

34. Dimitrios Katsaros and Pavlos Basaras. Detecting Influential Nodes in Complex Networks with Range Probabilistic Control Centrality, pages 265-272. Springer International Publishing, Cham, 2015.

35. J. Kangasharju, J. Ott, and O. Karkulahti. Floating content: Information availability in urban environments. In Pervasive Computing and Communications Workshops (PERCOM Workshops), 2010 8th IEEE International Conference on, pages 804-808, March 2010. 